\title{
Transformation of NIH3T3 mouse fibroblast cells by MUC16 mucin (CA125) is driven by its cytoplasmic tail
}

\author{
PANAGIOTA GIANNAKOUROS, ISABELLE MATTE, CLAUDINE RANCOURT and ALAIN PICHÉ \\ Département de Microbiologie et Infectiologie, Faculté de Médecine, \\ Université de Sherbrooke, Sherbrooke, Québec J1H 5N4, Canada
}

Received May 8, 2014; Accepted June 26, 2014

DOI: $10.3892 /$ ijo.2014.2707

\begin{abstract}
MUC16 (CA125) is a transmembrane mucin that contributes to the progression of epithelial ovarian cancer (EOC). Expression of MUC16 is not detectable in the epithelial surface of normal ovaries. MUC16 expression is, however, common in serous EOC as well as in metastatic and recurrent tumors. Despite these observations, its contribution to the development of EOC is unknown. We stably expressed either empty vector, MUC16 C-terminal domain (MUC16 CTD) or MUC16 TMU (a construct that lacks the cytoplasmic tail) in NIH3T3 mouse fibroblast cells. In this study, we provide evidence for the role of MUC16 CTD in oncogenic transformation. We show that ectopic expression of MUC16 CTD enhances the growth of NIH3T3 cells under normal and low serum conditions, and promotes anchorage-dependent colony formation. The deletion of the cytoplasmic tail abrogated these effects. MUC16 CTD expression in NIH3T3 cells also enhances the formation of colony in soft agar as compared to MUC16 TMU. MUC16 CTD expression enhances tumor formation in nude mice. Our findings provide the first evidence that MUC16 induces the transformation of NIH3T3 cells and indicate that MUC16 functions as an oncogene. Furthermore, our data suggest that the cytoplasmic tail is critical for MUC16 oncogenic properties.
\end{abstract}

\section{Introduction}

MUC16 mucin (CA125) is overexpressed in the majority of high grade serous ovarian carcinomas (HGSOC) but not by normal ovarian surface epithelial (OSE) cells (1-7). In addition, the transformation of OSE cells has been associated with expression of MUC16 at high levels $(8,9)$. Rising and falling levels of serum MUC16 correlate with ovarian cancer (OC) progression and regression, making MUC16 the single most important OC clinical biomarker $(10,11)$. MUC16 mucin is a high molecular weight glycoprotein $(200-2000 \mathrm{kDa})$ that

Correspondence to: Dr Alain Piché, Département de Microbiologie et Infectiologie, Université de Sherbrooke, 3001, 12ième Avenue Nord, Sherbrooke, Québec J1H 5N4, Canada

E-mail: alain.piche@usherbrooke.ca

Key words: MUC16, mucin, ovariancancer, transformation, NIH3T3 shares many characteristics of the membrane-bound mucin proteins (12-20). MUC16 is type I transmembrane mucin with a large $\mathrm{N}$-terminal domain (>12000 amino acid residues) that is heavily glycosylated. The MUC16 extracellular central domain contains up to 60 glycosylated tandem repeated sequences (156 amino acids), a characteristic of mucins. The extracellular portion of MUC16 is shed after proteolytic cleavage and found in serum of women with HGSOC. The C-terminal domain (CTD) is composed of an extracellular region with potential proteolytic cleavage sites, a transmembrane domain and a short 31 amino acid cytoplasmic tail (CT) (13-15). The phosphorylation of MUC16 CT stimulates its cleavage and release into the serum (21). MUC16 CT, which shares no homology with other transmembrane mucins CT, contains a highly conserved RRRKK amino acid polybasic sequence acting as a ezrin/radixin/moesin (ERM) family binding site (12-15).

Despite its clinical importance as a serum biomarker for OC, its role in the pathogenesis of OC has not been well characterized. The large extracellular domain appears to play a significant role in determining the adhesive properties of tumor cells. MUC16 binds to galectin-1 protein (22). Although the biological significance of this interaction remains unclear, the cell-surface recruitment of galectin-1 has been associated with processes such as regulation of cell adhesion (23). MUC16 facilitates heterotypic cell adhesion through its binding with mesothelin (24,25). Mesothelin-MUC16 interaction could facilitate heterotypic cell-cell adhesion and peritoneal metastasis of ovarian tumors through the adhesion with mesothelial cells. Patankar et al reported that natural killer (NK) cells incubated with soluble MUC16 exhibited a 50-70\% decrease in the lysis of tumor cells (26). MUC16-expressing EOC cells are also protected from lysis by primary NK cells (27). MUC16 downregulates CD16 expression in NK cells found in peritoneal fluids of patients with EOC (28). Secreted MUC16 binds to NK cells, B cells and monocytes via Siglec-9, a receptor found on immune cells that inhibits the NK cell response (28). The high levels of secreted MUC16 found in ascites may be one of the factors contributing to the immunosuppressive properties of ascites. The function of the MUC16 CT is not known. Ectopic expression of MUC16 CTD has been associated with the attenuation of drug-induced apoptosis in EOC cells $(30,31)$. Moreover, MUC16 CTD expression increases the tumorigenicity of SKOV3 cell line in a xenograft mouse model when compared to a similar construct lacking the CT 
domain (32). These data suggest a potential role of MUC16 CT in tumorigenesis.

In the present study, MUC16 CTD or a similar construct lacking the CT domain (MUC16 TMU) were stably expressed in mouse fibroblast NIH3T3 cells by lentiviral infection and their effects were assessed using in vitro and in vivo functional assays. We demonstrate that ectopic expression of MUC16 CTD, when compared to MUC16 TMU, enhances anchoragedependent and -independent growth and colony formation as well as tumorigenesis in nude mice, the hallmarks of malignant transformation. Together, these observations support the oncogenic potential of MUC16 and suggest that the CT domain is essential.

\section{Materials and methods}

Cell culture and reagents. The immortalized non-tumorigenic mouse fibroblast NIH3T3 cell line was obtained from the American Type Culture Collection (Rockville, MD). NIH3T3 cells were grown in DMEM (Wisent, St-Bruno, QC, Canada) supplemented with $10 \%$ FBS (Wisent), $2 \mathrm{mM}$ L-glutamine (Wisent), $100 \mathrm{U} / \mathrm{ml}$ penicillin and $100 \mu \mathrm{g} / \mathrm{ml}$ streptomycin. 293T cells were cultured in DMEM (Wisent) supplemented with 10\% FBS, $0.1 \mathrm{mM}$ MEM non-essential amino acid solution and $10 \mathrm{mM}$ HEPES. Cells were maintained at $37^{\circ} \mathrm{C}$ in humidified $5 \% \mathrm{CO}_{2}$ incubators. MUC16 C-terminal domain (MUC16 CTD) (containing last $284 \mathrm{C}$-terminal residues) was amplified by PCR from pME18S-FLJ14303 (Helix Research Institute, Japan). The 5' primer integrated an EcoRV restriction site while the $3^{\prime}$ primer integrated a XhoI restriction site. The digested amplicon was inserted in a pLenti6V5 vector containing at the $5^{\prime}$ end an IgK leader sequence, a myc-tag and a small linker (23 residues) and at the 3 ' end a linker sequence and a Flag-tag (21 residues) $(33,34)$. The control vector lacked the amplified MUC16 CTD sequence. MUC16 TMU contains the extracellular unique region and the transmembrane domain. To ensure anchorage of the construction at the cytoplasmic membrane, three residues adjacent to the transmembrane domain in the cytoplasmic tail were conserved in MUC16 TMU. After verification by sequencing, 293T cells were transfected with equal amout of pLenti vectors Plp1, Plp2, PlpVSVG and either the pLenti6V5-MUC16-CTD, -EV or -MUC16 TMU using Lipofectamine 2000 (Life Technologies, Burlington, ON, Canada). Lentivirus produced by $293 \mathrm{~T}$ cells were collected 48 -h post-transfection and kept at $-80^{\circ} \mathrm{C}$. NIH3T3 cells were plated in 6-well plates at $90 \%$ confluence in complete medium DMEM. Polybrene $(4 \mu \mathrm{g} / \mathrm{ml}$ final concentration) was added to the vial of thaw lentiviruses. This mixture was incubated with the cells for $1 \mathrm{~h}$ at $37^{\circ} \mathrm{C}, 5 \% \mathrm{CO}_{2}$. After the initial incubation with the viruses, complete medium supplemented with polybrene (without antibiotics) was added. Forty-eight hours later the culture media was replaced with complete media containing blasticidin $(0.5 \mu \mathrm{g} / \mathrm{ml})$ (Sigma Canada, Ltd., Oakville, ON, Canada). NIH3T3 expressing either empty vector, MUC16 TMU or MUC16 CTD populations were obtained while maintaining the blasticidin selection throughout. Validation of expression for the populations was done by RT-PCR and WB analysis. Independent clones for MUC16 CTD-expressing NIH3T3 cells were obtained by limiting dilution in a 96-well plate with blasticidin selection.
$R T-P C R$. Primers for PCR amplification of MUC16 CTD were as follow: 5'-CAACTCGAGTTGCAGATCCTCCAGGTC TAG-3' (sense) and 5'-AACGATATCACCCTGCTGAGGGAC ATC-3' (antisense). Primers for PCR amplification of MUC16 TMU were as follow: 5'-AATATCTTAAGCGGCCGCACCG GGTGGTCACCAGGAC-3' (sense) and 5'-ATGCGGCCAT CTAGGCCACATCACCCTGAGGGAC-3' (antisense) (Integrated DNA Technologies, Inc., Coralville, IA). PCR was performed as follows: $94^{\circ} \mathrm{C}$ for $5 \mathrm{~min}$ followed by 30 cycles of $94^{\circ} \mathrm{C}$ for $1 \mathrm{~min}, 65^{\circ} \mathrm{C}$ for $1 \mathrm{~min}$ and $72^{\circ} \mathrm{C}$ for $1 \mathrm{~min}$ and finalized at $72^{\circ} \mathrm{C}$ for $15 \mathrm{~min}$.

Immunoblot analysis. Cell were lysed in lysis buffer $(50 \mathrm{mM}$ Tris- $\mathrm{HCl}$, pH 7.5, 0.1\% SDS, $150 \mathrm{mM} \mathrm{NaCl}, 0.5 \mathrm{mM}$ EGTA, $1 \%$ Triton $\mathrm{X}-100)$ with freshly added protease inhibitors [1 $\mu \mathrm{g} / \mathrm{ml} 4$-(2-aminoethyl) benzenesulfonyl fluoride hydrochloride, $2 \mu \mathrm{g} / \mathrm{ml}$ aprotinin, $0.7 \mu \mathrm{g} / \mathrm{ml}$ pepstatin and $0.5 \mu \mathrm{g} / \mathrm{ml}$ leupeptin] (Sigma) and proteins were quantified by Bradford assay (Bio-Rad, Mississauga, ON, Canada).

Cells lysates (equal amounts of proteins) were submitted to SDS-PAGE electrophoresis (10\%) and transferred onto PVDF membrane (Roche, Laval, QC). The membranes were blocked with $5 \%$ non-fat milk and probed with indicated antibodies. Anti-Flag M2 antibody (1:500) and anti- $\alpha$-tubulin (1:25000) antibodies were purchased from Sigma. Anti-c-myc sc-789 antibody (1:1000) and anti-ERK 1: sc-93 (1:250) were from Santa Cruz Biotechnology (Dallas, TX, USA). Anti-phospho-p44/42

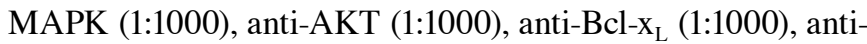
cyclin A2 (1:1000), anti-cyclin D1 (1:1000), anti-cyclin D2 (1:1000), anti-cyclin E2 (1:1000) and HRP-conjugated rabbit anti-mouse antibody and anti-rabbit antibody were all from Cell Signaling Technology (Danvers, MA, USA). Anti-Mcl1 (1:500) was purchased from BD Bioscience (Mississauga, ON, Canada). Anti-Bcl-2 (1:1000) antibody was from Dako (Burlington, ON, Canada). Anti-phospho-Akt (Ser 473) was from Life Technologies. The immunoblots were developed with chemiluminescence using the ECL system according to the manufacturer's instruction (GE Healthcare, Baie d'Urfe, QC).

Soft agar colony formation. Cells $\left(1 \times 10^{5}\right)$ were suspended in complete medium containing $0.33 \%$ of melted bactoagar. The resulting suspension was added to a $60-\mathrm{mm}$ plate covered with a $2-\mathrm{ml}$ layer of solidified $0.66 \%$ bacto-agar in complete medium. Grossly visible colonies were stained with MTT [3-(4,5-dimethylthiazol-2-yl)-2,5-diphenyltetrazolium bromide] (Sigma) and counted after 30 days of growth at $37^{\circ} \mathrm{C}$ in $5 \% \mathrm{CO}_{2}$ incubators. Three experiments were performed in triplicate. The mean \pm SD was calculated from three independent experiments.

Immunofluorescence. NIH3T3 clones were grown on glass slides until a $50-70 \%$ confluence was reached. Glass slides were then washed in cold PBS and cells fixed in $3.7 \%$ formaldehyde (Fisher) for $10 \mathrm{~min}$. Glass slides were next rinsed for $5 \mathrm{~min}$ in PBS and quenched (glycin $0.1 \mathrm{M}$ ) during $30 \mathrm{~min}$. Depending on the experiment, cells were permeabilized in PBS containing $0.2 \%$ Triton $\mathrm{X}-100$ for $20 \mathrm{~min}$ at room temperature. Slides were rinsed twice in PBS and blocked overnight in $\mathrm{PBS} / 2 \%$ goat serum at $4^{\circ} \mathrm{C}$. Slides were washed 3 times in PBS, incubated with primary anti-c-myc sc-789 
A

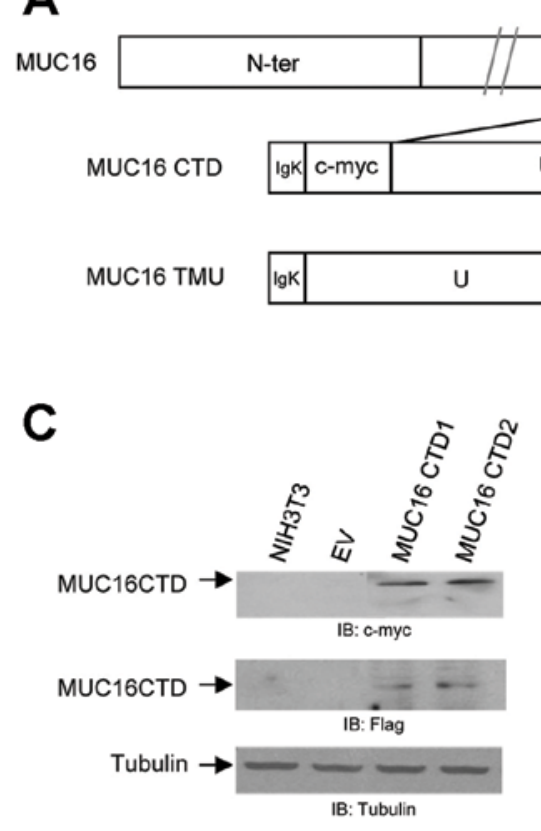

B

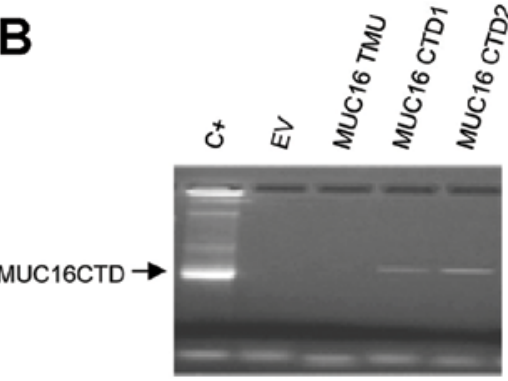

D
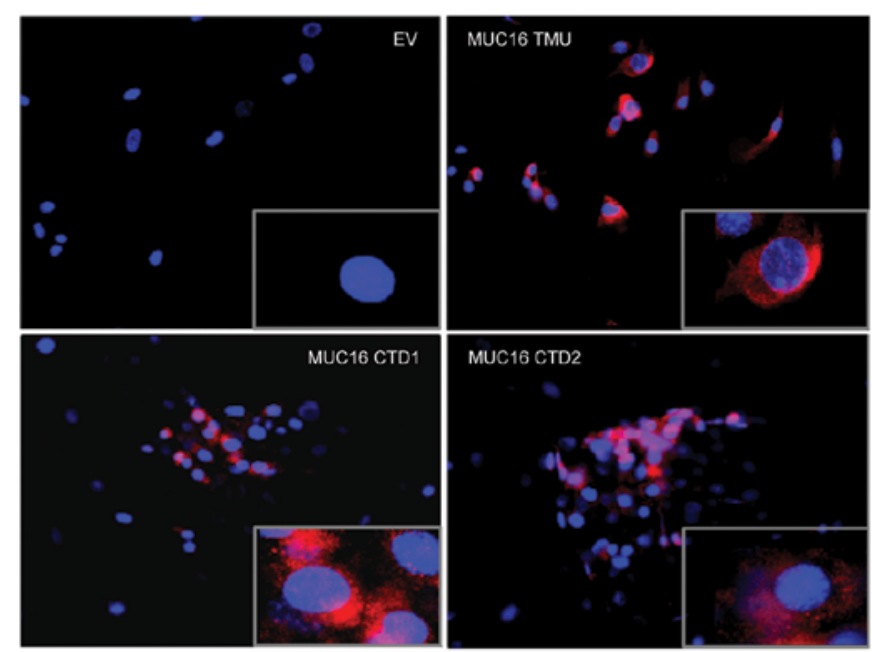

Figure 1. Expression of MUC16 CTD in NIH3T3 cells. (A) Schematic representation of MUC16, MUC16 CTD and MUC16 TMU constructs. The major domains of MUC16 include the N-terminal domain (N-ter), the tandem repeat domain (TR) and the C-terminal domain. The extracellular larger subunit consists of the N-terminal (>12000 a.a.) and tandem repeat domains (156 a.a. each), and are heavily glycosylated. The smaller C-terminal domain contains a unique extracellular domain (U), a transmembrane domain (TM) and the cytoplasmic tail of 31 amino acids (CT). MUC16 CTD consists of the extracellular unique region (U), the transmembrane domain (TM) and the 31 amino acid cytoplasmic tail (CT). The construct also contains an IgK leader sequence to target the construct to the secretory pathway in order to anchor the protein at the cell surface. The $\mathrm{N}$-terminal is tagged with c-myc epitope and the C-terminal with Flag epitope to facilitate detection. MUC16 TMU is similar to MUC16 CTD except that the CT domain has been deleted. This construct also possesses a c-myc and a His at the C-terminal. (B) Detection of MUC16 CTD by RT-PCR in NIH3T3 cells. Total RNA from parental NIH3T3 cells, empty vector (EV)-, MUC16 CTD clone 1 (CTD1)- and clone 2 (CTD2)-expressing NIH3T3 cells was reverse transcribed and assayed by PCR. $\mathrm{C}^{+}$is from MUC16 CTD-expressing SKOV3 cells (34). (C) Expression of MUC16 CTD by immunoblot using anti-c-myc and anti-Flag antibodies. (D) Expression of MUC16 CTD and MUC16 TMU by immunofluorescence. Cells were grown on coverslips, fixed and non-permeabilized cells were stained with anti-c-myc antibody.

(1:200) antibodies in blocking buffer at room temperature for $1 \mathrm{~h}$. Slides were washed 3 times in PBS, incubated for $45 \mathrm{~min}$ at room temperature with Alexa FluoR 594 or F(ab')2 fragment of goat anti-mouse or goat anti-rabbit IgG from Life Technologies. After washing, slides were incubated for 2 min in 4',6-diamidino-2-phenylindole (DAPI) to visualize nuclei, washed again in PBS and mounted for visualization by fluorescence microscopy with an Olympus IX70 (Olympus, Hamburg, Germany). Expression of MUC16 CTD was detected using anti-c-myc sc-789 antibody (1:200).

Cell proliferation assay. Cell growth was determined by XTT assay. Briefly, cells were resuspended in complete medium and plated into 96-well plates at a density of $1 \times 10^{4}$ cells/well. Cells were cultured for various times in complete and low serum concentration before adding XTT reagents. After incubating for $1 \mathrm{~h}$ at room temperature, the absorbance was read at $450 \mathrm{~nm}$ using a microplate reader at $450 \mathrm{~nm}$ (Tecan Sunrise, Research Triangle Park, NC, USA). All assays were performed in triplicate and mean \pm SEM was calculated from three independent experiments.
Anchorage-dependent clonogenic assay. Cells were seeded in 6-well plates in complete medium. Fourteen days post-confluence, the cells were fixed and stained with crystal violet (Sigma) and the number of colonies composed of $>50$ cells was determined. All assays were performed three times. The mean \pm SEM was calculated from three independent experiments.

Spheroid formation. A drop of $15 \mu 1$ medium containing $5 \times 10^{3}$ cells was pipetted onto the inner surface of a Petri dish lid. The lid was then placed on the Petri dish so that the drops were hanging from the lid with the cells suspended within them. To eliminate evaporation, $10 \mathrm{ml}$ PBS was placed in the bottom of the Petri dish. During 5 days of incubation at $37^{\circ} \mathrm{C}$ in $5 \% \mathrm{CO}_{2}$ incubators, the lid of the Petri dish was inverted and photographed using a microscope at x100 magnification.

Tumorigenicity assay. Mice (athymic nu/nu) were purchased from Charles River (St-Constant, QC, Canada) and housed in pathogen-free animal facilities. Mice were treated according to the Institution Animal Care guidelines. To assess tumorigenic capacity of EV-, MUC16 CTD- and MUC16 TMU-expressing 

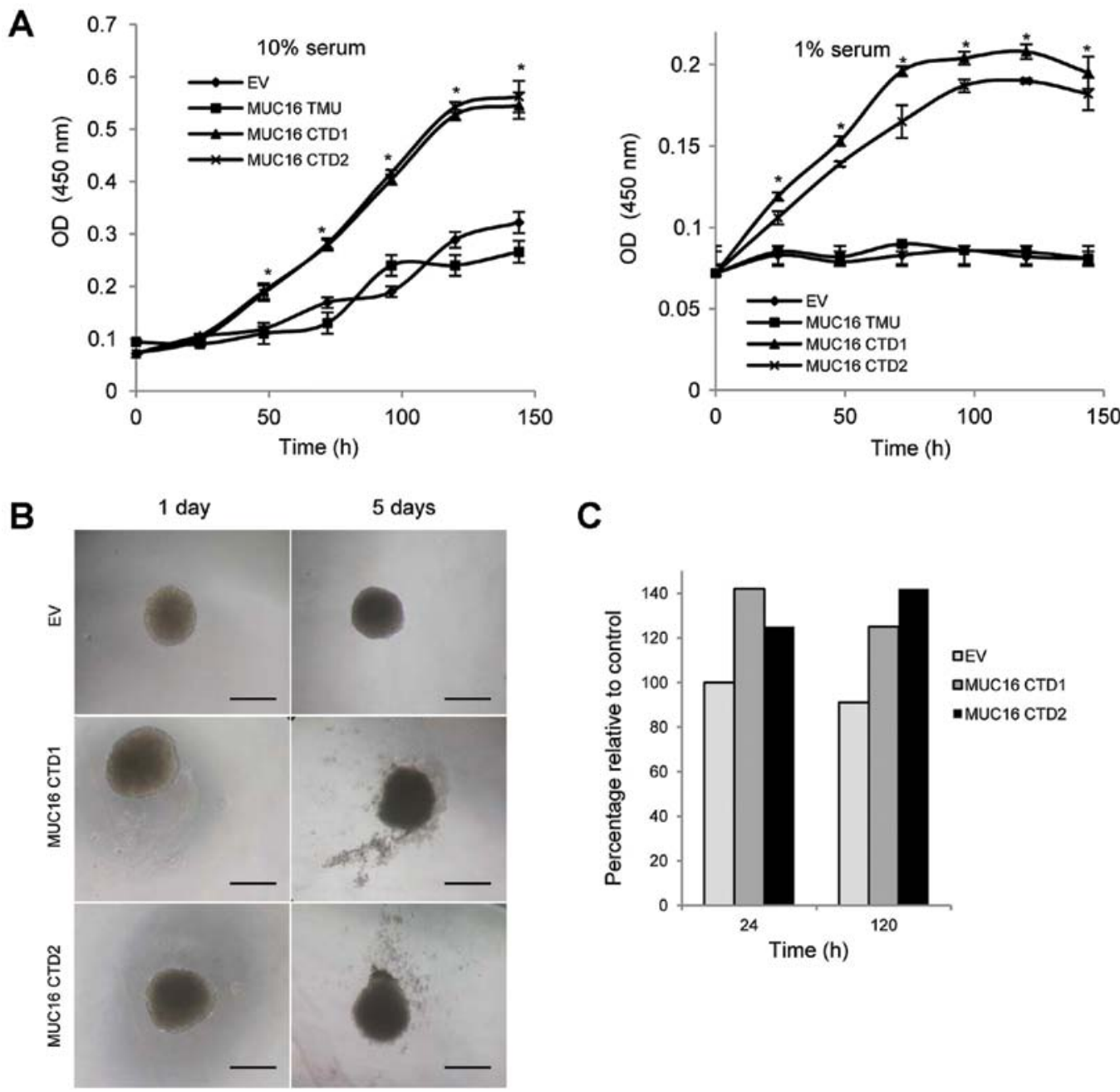

Figure 2. MUC16 CTD promotes anchorage-dependent growth. MUC16 CTD1- and MUC16 CTD2-expressing NIH3T3 cells and their control cells (MUC16 TMU and EV NIH3T3 cells) were used to assess the effect of MUC16 CTD on cell proliferation. (A) Cell proliferation was measured by XTT assay at an optical density (OD) absorbance of $450 \mathrm{~nm}$ and expressed as the mean $\pm \mathrm{SD}$. Data were from three independent experiments in triplicate. ${ }^{*} \mathrm{P}<0.001$ relative to controls. (B) Representative pictures of spheroids at Days 1 and 5. Scale bars, $200 \mu \mathrm{m}$. (C) The diameter was determined and expressed as the percentage of EV-expressing NIH3T3 cells assessed at $24 \mathrm{~h}$ (control).

NIH3T3 cells, $5 \times 10^{6}$ cells were suspended in serum-free medium and injected subcutaneously into 6-8 weeks old female athymic CD-1 nude mice. Each mouse received 4 injections in the flanks and each group contained 2 mice. Tumors were excised and weighted after 23 days. The mean tumor weight \pm SEM was calculated from eight independent site injections.

Statistical analyses. Statistical analysis was done using the BMDP statistical analysis software (UCLA, Los Angeles, CA). The threshold for statistical significance is a probability of 0.05 . In the nude mouse model, data were expressed as mean \pm SEM of tumor weight. The two-sided Student's t-test was used to assess statistical differences between groups for the in vivo mouse models.

\section{Results}

Expression of MUC16 constructs in NIH3T3 mouse fibroblast cells. To assess the potential of MUC16 in mediating oncogenic transformation, we infected the MUC16 negative immortalized non-tumorigenic NIH3T3 mouse fibroblast cell line with lentiviruses to generate NIH3T3 cell lines expressing empty vector (EV), MUC16 TMU or MUC16 CTD. MUC16 CTD consists of the last C-terminal 283 residues which include the transmembrane domain (TM) and cytoplasmic tail (CT) (Fig. 1A). Because of the lack of available antibody against MUC16 CTD, the construct was tagged with c-myc epitope at the $\mathrm{N}$-terminus and a Flag epitope at the $\mathrm{C}$-terminus (Fig. 1A). MUC16 TMU is similar to MUC16 CTD except that the CT domain was deleted (Fig. 1A). Stable clones were screened for MUC16 CTD expression and two independent clones that exhibited MUC16 CTD expression were selected (CTD1 and CTD2). Empty vector and MUC16 TMU consisted of pooled populations. The expression of MUC16 CTD in NIH3T3 cells was validated by RT-PCR (Fig. 1B) and immunoblotting with anti-c-myc and anti-Flag antibodies (Fig. 1C). The expression of MUC16 CTD and MUC16 TMU was further characterized by immunofluorescence with anti-c-myc antibody on nonpermeabilized cells (Fig. 1D).

MUC16 CTD enhances anchorage-dependent cell growth and promotes growth in low serum dependence. One of the 
A
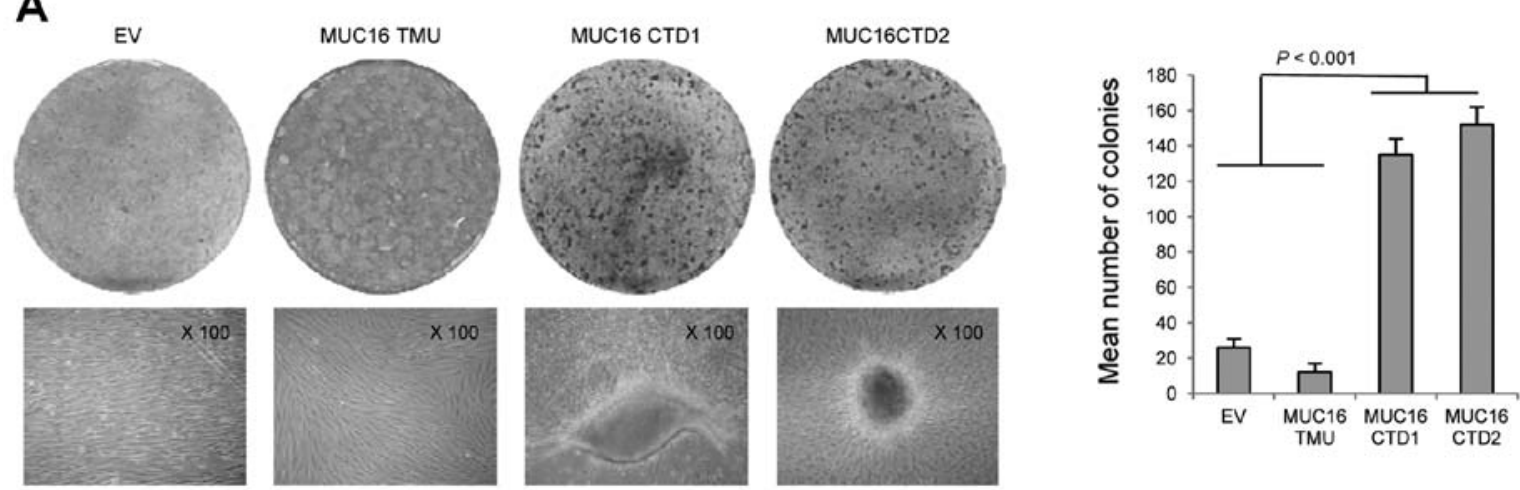

B
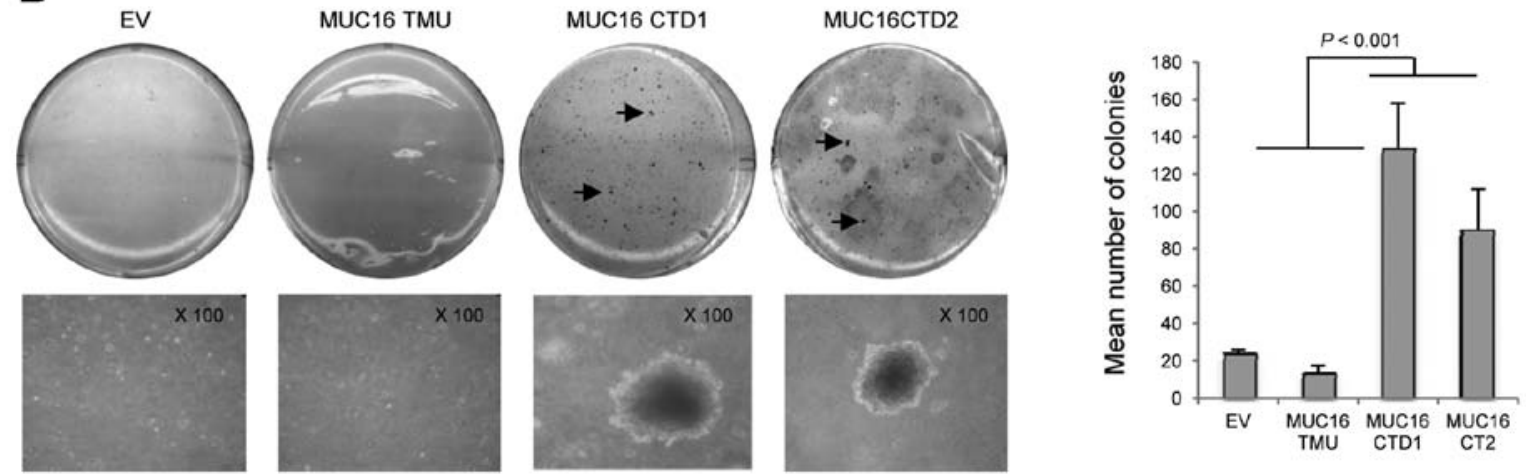

Figure 3. MUC16 CTD promotes colony formation under anchorage-dependent and -independent conditions. (A) Colony formation assay under anchoragedependent conditions was peformed by seeding $1 \times 10^{4}$ cells in 6-well plates and incubating cells for 2 weeks. Representative phase-contrast pictures of colonies after 2 weeks (magnification, x100). Colonies were counted and expressed as the number of colonies (mean \pm SD) per 6 -well plates from three independent experiments. (B) NIH3T3 cells expressing either EV, MUC16 TMU, MUC16 CTD1 and MUC16 CTD2 were suspended in soft agar and incubated for 3 weeks. Representative photomicrographs at $\mathrm{x} 100$ magnification are included. Colonies were counted and the results were expressed as the number of colonies $($ mean $\pm \mathrm{SD})$ from three independent experiments.

hallmarks of transformed cells is their ability to grow in the absence of exogenous factors, which is shown by the ability to grow in low serum conditions (35). To assess the effects of MUC16 expression on cell growth, EV-, MUC16 TMU and MUC16 CTD-expressing NIH3T3 cells were seeded at low density and the number of viable cells was assessed at different time intervals for up to 6 days in normal (10\%) and low (1\%) serum concentration (Fig. 2A). Expression of MUC16 CTD in both MUC16 CTD1 and MUC16 CTD2 led to significantly accelerated cell growth under both normal (10\%) and low (1\%) serum conditions when compared to cells expressing the empty vector or MUC16 TMU.

To further determine growth-related features, we assessed the capacity of cells to form three-dimensional structures using the hanging droplets technique (36). Larger spheroids were observed for MUC16 CTD-expressing NIH3T3 at Days 1 and 5 when compared to EV-expressing cells (Fig. 2B and C). EV-expressing NIH3T3 cells were able to form very compact spheroids whereas spheroids formed by MUC16 CTD-expressing cells were not as compact with cells detaching from the spheroid.

MUC16 CTD induces colony formation under anchoragedependent and anchorage-independent conditions. The effects of MUC16 expression on colony formation was assessed under anchorage-dependent and anchorage-independent conditions. Cells were seeded at low density and after 2 weeks (post-confluence), they were fixed and stained with crystal violet (Fig. 3A). MUC16 CTD-expressing NIH3T3 cells were characterized by loss of contact inhibition, overlapping growth and showed significantly $(\mathrm{P}<0.001)$ enhanced foci formation when compared to MUC16 TMU-transfected cells. Anchorage-independent growth was assessed by the formation of colony in soft agar. Under these conditions, there were few colonies with NIH3T3 cells expressing the empty vector or MUC16 TMU (Fig. 3B). NIH3T3 cells have been reported to occasionally spontaneously transform (37). More importantly, the number of soft agar colonies was markedly $(\mathrm{P}<0.001)$ increased with NIH3T3 cells expressing MUC16 CTD when compared to MUC16 TMU or EV-expressing cells. These data suggest that MUC16 CTD expression induces the transformation of NIH3T3 cells.

MUC16 CTD induces tumorigenicity. To further assess the oncogenic potential of MUC16 CTD expression, we examine the formation of subcutaneous tumors in mice injected with MUC16 CTD and MUC16 TMU. Because MUC16 CTD1 and MUC16 CTD2 expression resulted in similar phenotype in vitro, only MUC16 CTD1-expressing cells were further selected for in vivo experiments. Mice injected with MUC16 CTD1-expressing NIH3T3 cells grew much larger tumors over time when compared to MUC16 TMU-expressing cells (Fig. 4A and B). The average tumor volume in NIH3T3 
A

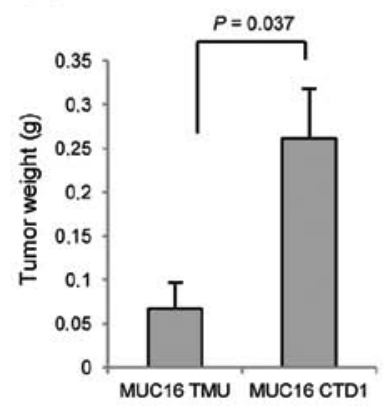

B

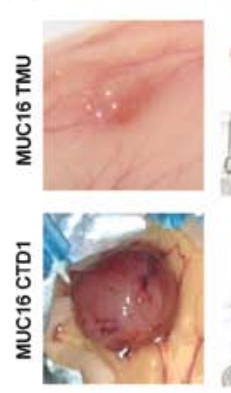

C

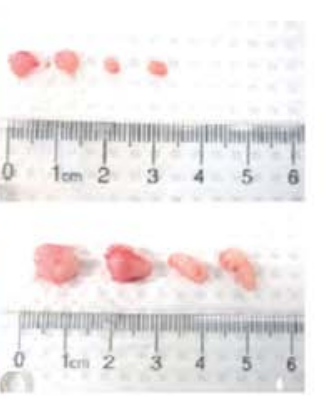

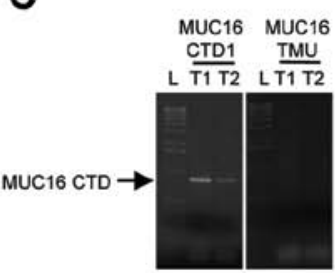

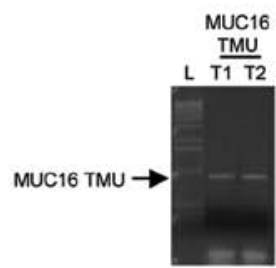

Figure 4. MUC16 CTD induces tumorigenicity of NIH3T3 in nude mice. (A) MUC16 TMU- and MUC16 CTD1-expressing NIH3T3 cells (5x106) were injected subcutaneously into the flank of nude mice. After 23 days, tumors were excised and weighed. Results were expressed as tumor weight (mean \pm SD) from experiments that included 2 mice with 4 injections/mice/group. (B) Representative subcutaneous tumors formed by MUC16 TMU and MUC16 CTD1expressing NIH3T3 cells. Photos were taken on Day 23 post-injection. (C) The expression of MUC16 TMU and MUC16 CTD1 in tumors was evaluated by semi-quantitative RT-PCR using either primers specific to MUC16 CTD (left panel) or MUC16 TMU (right panel) in two independent subcutaneous tumors.

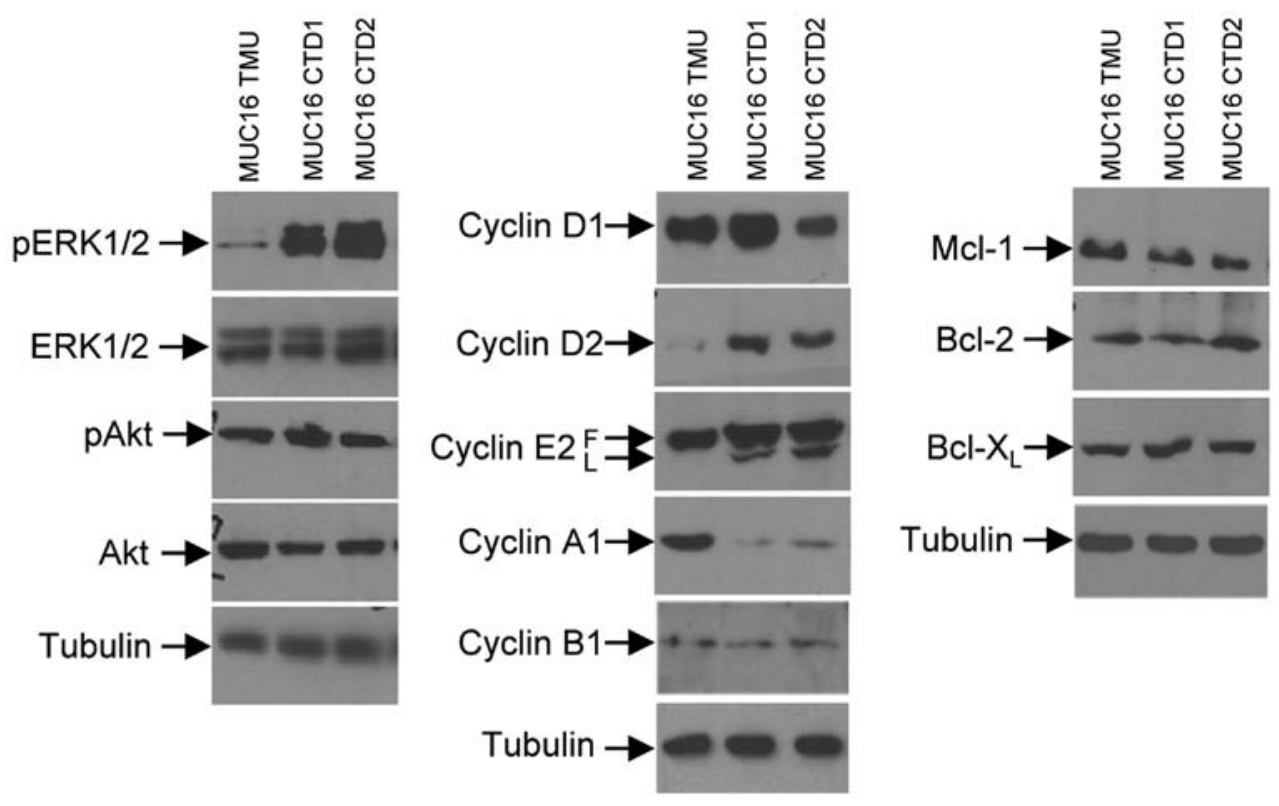

Figure 5. Immunoblot analysis of whole lysates from NIH3T3 cells overexpressing either MUC16 TMU, MUC16 CTD1 or MUC16 CTD2. Equal amount of proteins were loaded in each lane. F, full length form of cyclin E; L, low molecular weight form of cyclin E.

MUC16 CTD1 cell-injected mice exceeded $0.25 \mathrm{~g}$ at 23 days. In contrast, tumors from NIH3T3 MUC16 TMU-injected mice were $0.067 \mathrm{~g}$ at 23 days. The expression of MUC16 CTD and MUC16 TMU in the subcutaneous tumors was confirmed by RT-PCR (Fig. 4C).

MUC16 CTD expression and cell proliferation markers. MUC16 CTD expression increases NIH3T3 cell proliferation in vitro (Fig. 2) and in vivo (Fig. 4). We used immunoblot analysis to investigate the association between MUC16 CTD expression and the corresponding changes in the expression of proteins that are involved in cell cycle regulation, including ERK1/2, Akt, cyclin D1, cyclin D2, cyclin E and cyclin A. NIH3T3 cells expressing MUC16 CTD showed increase phosphorylation of the cell cycle regulatory protein ERK1/2 and had higher levels of G1-S cell cycle transition regulatory cyclin D2 and the short form of S-phase regulatory protein cyclin E that usually increases cell proliferation (Fig. 5). The presence of the shorter form of cyclin $\mathrm{E}$ is usually found in tumors of ovarian cancer cells, but not in normal cells (38). The levels of G2-M phase regulatory protein cyclin A1 was decreased whereas cyclin B1, another G2-M phase regulatory protein, was unchanged. The expression of proteins usually associated with cell survival such as Akt, Mcl-1, Bcl-2 and Bcl-X $\mathrm{L}_{\mathrm{L}}$ were unaffected by MUC16 CTD expression. Thus, MUC16 CTD expression is associated with increased expression of cell cycle regulatory proteins.

\section{Discussion}

MUC16 is emerging as a critical factor involved in different steps of OC oncogenesis. Ectopic expression of its C-terminal domain has been shown to confer resistance to genotoxic drugs such as cisplatin (30) and to death receptor-induced 
apoptosis (31). MUC16 CTD expression also enhances tumor cell growth, migration, invasion and metastasis of ovarian cancer cells (32). Furthermore, the epithelial-mesenchymal transition (EMT) of OC cells was shown to be regulated by MUC16 (39). Thus, MUC16 mucin is not only a useful clinical marker of disease progression and regression; it is also an important factor for the pathogenesis of OC. In this study, we further expand the functions of MUC16 by demonstrating that it induces oncogenic transformation of NIH3T3 cells. MUC16 CTD expression induces loss of contact inhibition, increases cell proliferation under normal and low serum conditions, enhances anchorage-independent growth and promotes tumorigenesis in vivo. These data suggest that, in addition to being important for ovarian cancer progression, MUC16 appears to play a role in early steps of cancer development. Interestingly, the acquired phenotypes of transformation induced by MUC16 CTD are quite similar to those reported with the expression of the C-terminal domain of other membrane-bound mucins such as MUC1 and MUC4 (40,41). MUC1 and MUC4 mucins have well established growth-promoting activity (42-44). Although the oncogenic function of MUC16, MUC1 and MUC4 appears to be located in their CTD, MUC16 CT shares no homology with MUC1 CT and MUC4 CT, suggesting that the underlying mechanisms for transformation differs between MUC16 and MUC1 and MUC4.

The detailed molecular mechanisms by which MUC16 CTD promotes oncogenic transformation of NIH3T3 remain unknown. The findings that expression of MUC16 CTD, but not MUC16 TMU, is sufficient for cell transformation indicate that intracellular signaling function of MUC16 CT is responsible for the transformed phenotype. We found that MUC16 CTD expression promoted cell proliferation which was associated with increased levels of proteins that controlled the cell cycle such as cyclin D2 and short cyclin E isoform. MUC16 CTD expression also resulted in the activation ERK1/2 signaling in NIH3T3 cells. In contrast, proteins associated with cell survival such Akt and anti-apoptotic proteins Bcl-2, Bcl- $\mathrm{X}_{\mathrm{L}}$ and Mcl-1 were not altered by MUC16 CTD expression. The activation of ERK1/2 signaling and the upregulation of cyclin D2 and cyclin E might be responsible for MUC16 CTD-induced NIH3T3 cell proliferation.

In this study, the oncogenic potential of MUC16 CTD was tested with a classical model; the NIH3T3 cells. However, it will be interesting to further determine whether MUC16 CTD expression can induce the transformation of ovarian surface epithelial (OSE) cells. Carcinomas arising from the ovarian surface epithelium or the related distal part of the fimbriae are by far the most common subtypes. The transformation of human OSE cells have been generally achieved using a set of combined genetic elements such as introducing SV40 $\mathrm{T}$ antigen, human telomerase reverse transcriptase (hTERT) and oncogenic KRAS $(45,46)$. Interestingly, transformed OSE cells often expressed MUC16 whereas normal OSE cells are usually negative for MUC16 expression (1). Experiments are ongoing in our laboratory to determine whether MUC16 CTD is able to transform human OSE cells. This model would be ideal for understanding the mechanims by which MUC16 CTD could induce transformation.

In conclusion, we have demonstrated, for the first time, the role of MUC16 in cellular transformation. We show that
MUC16 CTD results in enhanced cell growth in adherent and non-adherent conditions and induces tumor formation in vivo. These acquired phenotypes were associated with altered expression of cell cycle regulatory proteins. Although further validation is necessary, MUC16 may represent a target to prevent the development of ovarian cancer.

\section{Acknowledgements}

A.P. and C.R. are members of the FRSQ-funded Centre de Recherche Clinique du Centre Hospitalier Universitaire de Sherbrooke (CRCHUS). This study was supported by funds from the Canadian Institute for Health Research (MOP-244194CPT-CFDA-48852) to A.P. and funds from the Canadian Cancer Society to C.R. (No. 0112225 and No. 014263).

\section{References}

1. Auersperg N, Wong AS, Choi KC, et al: Ovarian surface epithelium: biology, endocrinology and pathology. Endocr Rev 22: 255-288, 2001.

2. Bast RC Jr, Feeney M, Lazarus H, et al: Reactivity of a monoclonal antibody with human ovarian carcinoma. J Clin Invest 68: 1331-1337, 1981.

3. Canney PA, Moore M, Wilkinson PM, et al: Ovarian cancer antigen CA125: a prospective clinical assessment of its role as a tumour marker. Br J Cancer 50: 765-769, 1984.

4. Kabawat SE, Bast RC, Welch WR, et al: Immunopathologic characterization of a monoclonal antibody that recognizes common surface antigens of human ovarian tumors of serous, endometrioid, and clear cell types. Am J Clin Pathol 79: 98-104, 1983.

5. Nap M, Vitali A, Nustad K, Bast RC Jr, O'Brien TJ, et al: Immunohistochemical characterization of 22 monoclonal antibodies against the CA125 antigen: 2nd report from the ISOBM TD-1 Workshop. Tumor Biol 17: 325-331, 1996.

6. Nustad K, Bast RC Jr, O'Brien TJ, et al: Specificity and affinity of 26 monoclonal antibodies against the CA125 antigen: first report from the ISOBM TD-1 workshop. International Society for Oncodevelopmental Biology and Medicine. Tumor Biol 17: 196-219, 1996.

7. Høgdall EV, Christensen L, Kjaer SK, et al: CA125 expression pattern, prognosis and correlation with serum CA125 in ovarian tumor patients: from the Danish MALOVA ovarian cancer study. Gynecol Oncol 104: 508-515, 2007.

8. Ong A, Maines-Bandiera SL, Roskelley CD and Auersperg N: An ovarian adenocarcinoma line derived from SV40/E-cadherintransfected normal human ovarian surface epithelium. Int J Cancer 85: 430-437, 2000.

9. Zheng J, Mercado-Uribe I, Rosen DG, et al: Induction of papillary carcinoma in ovarian surface epithelial cells using combined genetic elements and peritoneal microenvironment. Cell Cycle 9: 140-146, 2010.

10. Bast RC Jr, Klug TL, St John E, et al: A radioimmunoassay using a monoclonal antibody to monitor the course of epithelial ovarian cancer. N Eng J Med 309: 883-887, 1983.

11. Vergote IB, Børmer OP and Abeler VM: Evaluation of serum CA 125 levels in the monitoring of ovarian cancer. Am J Obstet Gynecol 157: 88-92, 1987.

12. Bafna S, Kaur S and Batra SK: Membrane-bound mucins: the mechanistic basis for alteration of the growth and survival of cancer cells. Oncogene 29: 2893-2904, 2010.

13. Yin BW and Lloyd KO: Molecular cloning of the CA125 ovarian cancer antigen: identification as a new mucin (Muc16). J Biol Chem 276: 27371-27375, 2001.

14. Yin BW, Dnistrian A and Lloyd KO: Ovarian cancer antigen CA125 is encoded by the MUC16 mucin gene. Int J Cancer 98: 737-740, 2002.

15. O'Brien TJ, Beard JB, Underwood LJ, et al: The CA125 gene: an extracellular superstructure dominated by repeated sequences. Tumour Biol 22: 348-366, 2001.

16. O'Brien TJ, Beard JB, Underwood LJ and Shigemasa K: The CA125 gene: a newly discovered extension of the glycosylated N-terminal domain doubles the size of this extracellular superstructure. Tumour Biol 23: 154-169, 2002. 
17. de los Frailes MT, Stark S, Jaeger W, et al: Purification and characterization of the CA 125 tumor-associated antigen from human ascites. Tumor Biol 14: 18-29, 1993.

18. Kobayashi H, Ida W, Terao T and Kawashima Y: Molecular characteristics of the CA 125 antigen produced by human endometrial epithelial cells: comparison between eutopic and heterotopic epithelial cells. Am J Obstet Gynecol 169: 725-730, 1993.

19. Fendrick JL, Staley KA, Gee MK, et al: Characterization of CA 125 synthesized by the human epithelial amnion WISH cell line. Tumor Biol 14: 310-318, 1993.

20. Nagata A, Hirota N, Sakai T, et al: Molecular nature and possible presence of a membranous glycan-phosphatidylinositol anchor of CA125 antigen. Tumor Biol 12: 279-286, 1991.

21. Fendrick JL, Konishi I, Geary SM, et al: CA125 phosphorylation is associated with its secretion from the WISH human amnion cell line. Tumor Biol 18: 278-289, 1997.

22. Seelenmeyer C, Wegehingel S, Lechner J and Nickel W: The cancer antigen CA125 represents a novel counter receptor for galectin-1. J Cell Sci 116: 1305-1318, 2003.

23. Perillo NL, Marcus ME and Baum LG: Galectins: versatile modulators of cell adhesion, cell proliferation, and cell death. J Mol Med 76: 402-412, 1998.

24. Rump A, Morikawa Y, Tanaka M, et al: Binding of ovarian cancer antigen CA125/MUC16 to mesothelin mediates cell adhesion. J Biol Chem 279: 190-198, 2004.

25. Gubbels JA, Belisle J, Onda M, et al: Mesothelin-MUC16 binding is a high affinity, $\mathrm{N}$-glycan dependent interaction that facilitates peritoneal metastasis of ovarian tumors. Mol Cancer 5: 50, 2006.

26. Patankar MS, Jing Y, Morrison JC, et al: Potent suppression of natural killer cell response mediated by the ovarian tumor marker CA125. Gynecol Oncol 99: 704-713, 2005.

27. Gubbels JA, Felder M, Horibata S, et al: MUC16 provides immune protection by inhibiting synapse formation between NK and ovarian tumor cells. Mol Cancer 9: 11, 2010.

28. Belisle JA, Gubbels JA, Raphael CA, et al: Peritoneal natural killer cells from epithelial ovarian cancer patients show an altered phenotype and bind to the tumour marker MUC16 (CA125). Immunol 122: 418-429, 2007.

29. Belisle JA, Horibata S, Jennifer GA, et al: Identification of Siglec-9 as the receptor for MUC16 on human NK cells, B cells, and monocytes. Mol Cancer 9: 118, 2010.

30. Boivin M, Lane D, Piché A and Rancourt C: CA125 (MUC16) tumor antigen selectively modulates the sensitivity of ovarian cancer cells to genotoxic drug-induced apoptosis. Gynecol Oncol 115: 407-413, 2009.

31. Matte I, Lane D, Boivin M, et al: MUC16 mucin (CA125) attenuates TRAIL-induced apoptosis by decreasing TRAIL receptor $\mathrm{R} 2$ expression and increasing c-FLIP expression. BMC Cancer 14: 234, 2014.
32. Thériault C, Pinard M, Comamala M, et al: MUC16 (CA125) regulates epithelial ovarian cancer cell growth, tumorigenesis and metastasis. Gynecol Oncol 121: 434-443, 2011.

33. Piché A, Grim J, Rancourt C, et al: Modulation of Bcl-2 protein levels by an intracellular anti-Bcl-2 single-chain antibody increases drug-induced cytotoxicity in the breast cancer cell line MCF-7. Cancer Res 58: 2134-2140, 1998.

34. Kasono K, Heike Y, Xiang J, et al: Tetracycline-induced expression of an anti-c-Myb single-chain antibody and its inhibitory effect on proliferation of the human leukemia cell line K562. Cancer Gene Ther 7: 151-159, 2000.

35. Hanahan D and Weinberg RA: Hallmarks of cancer: the next generation. Cell 144: 646-674, 2011.

36. Zietarska M, Maugard CM, Filali-Mouhim A, et al: Molecular description of a 3D in vitro model for the study of epithelial ovarian cancer (EOC). Mol Carcinog 46: 872-885, 2007.

37. Bromberg JF, Wrzeszczynska MH, Devgan G, et al: Stat3 as an oncogene. Cell 98: 295-303, 1999.

38. Bedrosian I, Lu KH, Verschraegen C and Keyomarsi K: Cyclin E deregulation alters the biologic properties of ovarian cancer cells. Oncogene 23: 2648-2657, 2004

39. Comamala M, Pinard M, Thériault C, et al: Downregulation of cell surface CA125/MUC16 induces epithelial-to-mesenchymal transition and restores EGFR signaling in NIH:OVCAR3 ovarian carcinoma cells. Br J Cancer 104: 989-999, 2011

40. Li Y, Liu D, Chen D, et al: Human DF3/MUC1 carcinomaassociated protein functions as an oncogene. Oncogene 22: 6107-6110, 2003

41. Bafna S, Singh AP, Moniaux N, et al: MUC4, a multifunctional transmembrane glycoprotein, induces oncogenic transformation of NIH3T3 mouse fibroblast cells. Cancer Res 68: 9231-9238, 2008.

42. Satoh S, Hinoda Y, Hayashi T, et al: Enhancement of metastatic properties of pancreatic cancer cells by MUC1 gene encoding an anti-adhesion molecule. Int J Cancer 88: 507-518, 2000.

43. Singh AP, Moniaux N, Chauhan SC, et al: Inhibition of MUC4 expression suppresses pancreatic tumor cell growth and metastasis. Cancer Res 64: 622-630, 2004.

44. Komatsu M, Jepson S, Arango ME, et al: Muc4/sialomucin complex, an intramembrane modulator of ErbB2/HER2/Neu potentiates primary tumor growth and suppresses apoptosis in a xenotransplanted tumor. Oncogene 20: 461-470, 2001.

45. Sasaki R, Narisawa-Saito M, Yugawa T, et al: Oncogenic transformation of human ovarian surface epithelial cells with defined cellular oncogenes. Carcinogenesis 30: 423-431, 2009.

46. Mullany LK, Fan HY, Liu Z, et al: Molecular and functional characteristics of ovarian surface epithelial cells transformed by KrasG12D and loss of Pten in a mouse model in vivo. Oncogene 30: 3522-3536, 2011. 